\title{
La ratio del bien. Comentario y traducción del capítulo III del libro II de la Teología platónica de Marsilio Ficino
}

\section{The Ratio of Good. Coment and Translation of Chapter III in Book II of Marsilio Ficino's Platonic Theology}

\author{
ERnesto Priani Saisó \\ Universidad Nacional Autónoma de México \\ epriani@gmail.com \\ AbSAlom García CHow \\ Universidad Nacional Autónoma de México \\ absalomg@hotmail.com
}

El objetivo principal de este artículo es presentar la traducción del capítulo III del libro II de la Teología platónica de Marsilio Ficino, aportando algunas notas sobre la forma de escribir de Ficino, que pueden servir como una primera base para acometer una traducción más amplia de la obra de este filósofo, así como discutir con detalle las implicaciones morales de la adopción de la tesis de la superioridad de la unidad sobre el ser, que es el contenido central del capítulo referido.

Nuestra conclusión es que la introducción del argumento de la eminencia de la unidad en la Teología platónica de Ficino conduce a un distanciamiento del modelo teleológico aristotélico-tomista, pues impide la asimilación de los fines de la vida a Dios como fin de la existencia humana. En síntesis, para Ficino el alma no puede ver al Uno como su propio fin, porque no se le presenta como constituido de algún elemento común con ella misma, por lo que la vida humana es una búsqueda constante por reencontrar el lazo que une las acciones de la vida con la razón de la unidad de lo que existe.

Palabras Clave: Ficino, unidad, almas

The main goal of this article is to present a translation of Chapter III of Book II of Ficino's Platonic Theology, providing some notes on the writing of Ficino, which can serve as a first basis to undertake a broader translation of the work of this philosopher, and to discuss in detail the moral implications of the adoption of the thesis of the superiority of unity over being, which is the central content of the chapter referred.

Our conclusion is that the introduction of the argument of the eminence of unity in Ficino's Platonic Theology leads to distance itself from the Aristotelian-Thomistic teleological model, since it prevents the assimilation of the ends of life to God as the end of human existence. In short, for Ficino the Soul cannot see the One as its own end, because it is not presented 
as being constituted of some common element with itself, so human life is a constant search to rediscover the bond that unites the actions of life with the reason of the unity of what exists.

KeYwORDS: Ficino, unity, soul

\section{INTRODUCCIÓN ${ }^{1}$}

En el capítulo III del libro II de la Teología platónica, aparece por primera vez en la obra del filósofo florentino Marsilio Ficino (1433-1499) la tesis de la superioridad de la unidad sobre el ser, que es esencial para entender muchos de los aspectos más polémicos y novedosos de su pensamiento.

La tesis es una interpretación de las dos primeras hipótesis del diálogo platónico Parménides, y tiene implicaciones directas en la comprensión de la naturaleza y función de la divinidad, en la afirmación de la inmortalidad del alma, en la concepción de la naturaleza humana y en la reflexión en torno a las funciones mediadoras en lo ontológico y en lo epistemológico del alma y la fantasía. Además, es el motivo de la segunda polémica entre Marsilio Ficino y Giovanni Pico della Mirandola, y de la redacción de los comentarios a los diálogos platónicos Parménides y Sofista, escritos por Ficino en distintos momentos de su vida (Allen, Icastes, 9 ss).

Con el comentario y la traducción al capítulo III del libro II de la Teología buscamos introducirnos en dos temas centrales en torno a la figura y el pensamiento de Ficino. Su concepción moral y el uso del latín.

Es bien sabido, desde la obra de referencia de Kristeller (Il pensiero, 311), que el florentino no tiene propiamente un sistema moral. Sin embargo, eso no quiere decir que en su obra no se encuentren elementos de reflexión que incidan en la moral y, en general, en la concepción de los fundamentos de una ética. En este sentido, consideramos que el argumento expuesto en el capítulo III, del libro II de la Teología, en el que Ficino presenta a la unidad no como fin del alma - en un sentido teleológico-, sino como parte del fundamento para cualquier clase de distinción temporal, cualitativa, ontológica o moral, tiene implicaciones de ruptura y de innovación no sólo en el terreno ontológico, sino particularmente en el terreno moral. Es más, se trata de una de las claves para entender el cambio de dirección de la discusión moral al abandonar el modelo teleológico de Aristóteles.

${ }^{1}$ El texto a partir del cual se traducen todos los pasajes de la Teología está tomado de Michel Allen, en Ficino, Platonic Theology, que a su vez toma como referencia la edición de Basilea de 1576 y la de Raymond Marcel de 1970.

Medievalia 50, 2018, pp. 247-260 
En lo que se refiere al latín, hay que señalar que son escasas las traducciones contemporáneas al español de la obra de Ficino y de la Teología en concreto, y más escasas las que se detienen en el estudio del latín ficiniano. ${ }^{2}$ Nuestra traducción se propone, al tiempo que ofrecer una versión al español de un capítulo clave de la Teología, aportar algunas notas sobre la forma de escribir de Ficino, que pueden servir como una primera base para acometer una traducción más amplia de la obra de este filósofo y un conocimiento mayor de su latín.

\section{ENTIS ATQUE UNIUS RATIO EST DIVERSA}

Para avanzar en la comprensión de la exposición de la tesis de la eminencia de la Unidad, es necesario, como primer paso, entender la naturaleza de la disputa y la ubicación del capítulo y del argumento todo dentro de la Teología platónica de Ficino.

La Teología platónica sigue, en cuanto a estructura, la forma medieval de la disputa que, en este caso tiene como centro la inmortalidad del alma. Así, la Teología está dividida en una consideración general sobre los grados del ser (libros I-IV), la argumentación de la inmortalidad con argumentos comunes (libro V), argumentos propios (libros VI-XII), las conclusiones (libros XIII-XIV) y la solución a las cuestiones (libros XV-XVIII) (Kristeller, Il pensiero, 23).

El capítulo que analizamos pertenece a la primera parte, es decir, a la exposición de los grados del ser. Pero no debemos perder de vista que en esa parte no se trata sólo de exponer la estructura de la jerarquía, sino que ésta ha de entenderse como resultado de la intención de mostrar "el carácter eminentemente humano de todo movimiento hacia el ser, que se origina en la inaceptable injusticia de la condición humana tal como se presenta en la experiencia inmediata" (Garín, Ficino, 80).

Por eso hay que partir, para el análisis, de considerar como referencia la ubicación, y la naturaleza de la sustancia intermedia de las cinco que

\footnotetext{
${ }^{2}$ En español existen dos versiones del Comentario al Banquete de Platón, una realizada por R. de la Villa para la editorial Tecnos, la otra hecha sobre una versión italiana del texto por Mariapía Lamberti y José Luis Bernal, para la Universidad Nacional Autónoma de México. Recientemente apareció una versión a uno de Los libros de la vida, 'De la vida sobria', editado por Luigi Cornaro, para la Asociación Española de Neuropsiquiatría. Ninguna de estas ediciones es bilingüe, ni tiene notas al texto latino.
}

Medievalia 50, 2018, pp. 247-260 
componen la estructura de lo real y que constituye el "dentro" de esa gradación de lo existente. Nos referimos al alma que, según la Teología, posee fundamentalmente dos cualidades: el movimiento y la pluralidad: "Además, el alma, porque es capaz de movimiento, de una cosa pasa hacia la otra. Por lo tanto, lo uno tiene en sí mismo lo otro y esta tiene una multitud. El alma es pues, en sí mismo, una multitud, una multitud, ciertamente, capaz de movimiento" (Ficino, Teol. Plat. I-VI, 2, 79). ${ }^{3}$

Pero es necesario destacar que Ficino supone que para que una cosa esté en movimiento, lo que no es (lo otro) es de alguna forma en aquello que se mueve. Es decir, que el no ser por ejemplo, gallina, está ya en el ser huevo. En otras palabras, que el binomio ser/no ser es irreducible al ser; lo que es tanto como afirmar que toda existencia involucra lo que es, al igual que aquello que no es. $Y$ es que en la medida en que Ficino comprende que el ser es doble, es decir, "el ser emerge como no ser", lo que llamamos ens primum (primer ser) es en sí mismo múltiple, pues en él, la razón de ser y la razón de ser uno, son distintas. Véase Ficino (Comentario, 238-240).

Lo señala Schiavone al presentar la Teología:

En el vocabulario ficiniano son frecuentes, de hecho, los términos ens, essentia, esse. Pero el alcance especulativo y el significado histórico de tal perspectiva se complica con la introducción de otro concepto, verdaderamente esencial: el de la unitas, por la cual, al lado de una ontología general de lo finito se pone una metafísica del absoluto a la luz de la relación dialéctica uno-múltiple. Como consecuencia, el concepto del unum supra ens o de la unitas como principium rerum implica la distinción entre la ratio ens y la ratio unitatis ("Introduzione", 4).

Y es esto lo que encontramos en la base del capítulo VI del libro I de la Teología, donde Ficino advierte que:

El ángel, que inmediatamente precede al alma, no puede ser una unidad inmóvil porque la distancia entre estas dos cosas particulares - la primera una pluralidad en movimiento, y la otra una unidad inmóvil — aparece inmensa. La unidad es, por supuesto, el opuesto de la pluralidad, y lo que es inmóvil de lo que es móvil. Pero como en todo estas dos se oponen una a la otra, no pueden

${ }^{3}$ Porro anima, quia mobilis est, ab alio pertransit in aliud. Igitur aliud in se habet et aliud. Quod haec habet et multitudinem. Quapropter anima in seipsa multitudo quaedam est, multitudo inquam mobilis.

El argumento se encuentra también en Proclo, Elementos de teología, cap. 20, p. 45: "porque la inteligencia ... no es todavía unidad ... es objeto de su propia actividad”.

Medievalia 50, 2018, pp. 247-260 
venir una después de la otra: necesitan un vínculo. El ángel pues, precede al alma, que es pluralidad en movimiento, sin intermediarios (I-VI, 2,79$){ }^{4}$

Luego entonces, el orden de procesión de todas las cosas se establece en la Teología, sobre la base de la distinción de las tres más eminentes, como un ascender del movimiento y la pluralidad, a la pluralidad inmóvil, y de ahí, a la unidad inmóvil.

El pasaje subraya la falta de elementos comunes entre el alma y la unidad, y tiene como consecuencia que el alma no pueda ver más allá del ángel y sólo pueda tener noticia de que hay algo más allá a través de él. Pero ese algo ¿es uno? O, si hay uno más, ¿no podría haber otra serie infinita?

\section{Si DII SIC INNUMERABILES SINT}

Los capítulos II y III del libro II están dedicados a enfrentar dos posibles implicaciones negativas de suponer que hay algo más allá de la pluralidad inmóvil del ángel que es a la que directamente tiene acceso el alma humana. Así, en el capítulo II Ficino discute qué pasaría si, en efecto, hubiera dos dioses iguales. Su intención, explícita al final del capítulo, es mostrar que la postulación de una unidad por encima del ángel no conduce ni supone la dualidad maniquea (II-II, 10, 107). ${ }^{5}$

Pero en el capítulo III, que es el que nos interesa aquí, Ficino se propone mostrar que la suposición de que hay un Dios por encima del ángel no implica la existencia de un dios sobre otro dios hasta el infinito. Ni, por lo tanto, que pueda ser infinita la procesión de las causas. Hay que añadir sólo que la preocupación de Ficino en este capítulo es la misma de Proclo en la primera parte de los Elementos de teología: que toda multiplicidad y mesura es posterior al uno.

\footnotetext{
${ }^{4}$ Angelus, qui proxime hanc antecedit, esse nequit immobilis unitas quia duae quaedam huiusmodi res, quarum una sit mobilis multitudo, et altera immobilis unitas, longissime inter se distare videntur, unitas siquidem multitudini opponitur, immobile mobili. Quoniam ergo res illae ab omniparte invecem opponuntur, proxime sibi non succedunt, sed medio quodam indigent copulante.

${ }^{5}$ El centro del argumento es simple: si hubiera dos dioses iguales estos serían, o completamente diferentes, o idénticos o parcialmente iguales. Desecha las primeras dos posibilidades porque, si fueran idénticos, sería el mismo, y si fueran completamente diferentes no podrían ser iguales. La tercera opción, el que fueran parcialmente similares, obliga, según Ficino, a asumir que tienen una naturaleza en común y, por tanto, que esa naturaleza en común es más alta que cualquiera de los dos principios que no serían simples sino compuestos - por la naturaleza común y la propia de cada uno-.
}

Medievalia 50, 2018, pp. 247-260 
El centro del argumento es que si pensamos que el número de las causas es infinito, tendríamos que suponer que el número de los efectos también lo es. "Si los dioses fueran infinitos, las causas de las cosas serían infinitas, y una causa estaría por encima de otra gradual y perpetuamente $[\ldots]$. De esta forma, también los efectos de estas causas en el mundo serían infinitos, ya que cualquier causa produce algún efecto en el mundo" (II-III, 2, 109). ${ }^{6}$

Pero esto es absurdo, según Ficino, porque "en las cosas no existiría ninguna secuencia” y el flujo de estas no podría ir de un punto hacia otro. De hecho, no habría manera de juzgar al alma mejor que el cuerpo, porque tanto una como otro distarían de la suma bondad un infinito y zcómo comparar infinitos?, ¿cuál sería su medida?

En concreto, el problema es que estaríamos ante un fluir de todo hacia todas partes, donde no habría unidad, igualdad, similitud, estabilidad, orden o restauración, nada que suponga una mesura, porque no habría referencia ni unidad para efectuarla. Estaríamos, pues, ante la imposibilidad de establecer ninguna distinción en el orden de las cosas y, en consecuencia, claro, en el orden de la vida moral, como se entiende implícitamente a través del ejemplo del cuerpo y el alma. En otras palabras, todas las cosas serían igualmente infinitas, y ninguna causa sería mejor que su efecto. Pero, dirá Ficino:

Si no existiera en las cosas un principio, cualquier cosa fluiría a partir de cualquier otra, y en consecuencia todas fluirían. Si esto fuera así, nunca existiría la unidad, la igualdad, la semejanza, la permanencia, el orden y la restitución. Sin embargo, porque estos atributos existen, es necesario que el flujo del cual manan las cosas sea conducido y contenido por un eje que no fluya (II-III, 4, 111).?

Para explicar el movimiento, es decir, para dar cuenta de la identidad de lo que cambia, e identificar una secuencia, es necesario que las cosas fluyan desde un punto que es emanado - la mente-, pero contenido por lo que es uno. Así, la razón de lo que fluye y de lo que permanece, son diferentes en las cosas animadas. Y es esta diferencia la que hace que sea el uno - y no el alma — la razón de la ordenación y de la secuencia, de su jerarquización y de su diferencia. Lo que

${ }^{6}$ Si dii sic innumerabiles sint, erunt rerum causae infinitae se gradatim innumere superantes [...] Si ita, erunt quoque ejfectus in mundo innumerabiles.

${ }^{7}$ Si non sit in rebus primum aliquid, res quaelibet emanabit ab alia; omnes igitur fluitabunt. Quapropter nunquam erit unitas, aequalitas, similitudo, status, ordo et restitutio. Nunc vero quia haec rebus insunt, oportet rerum ab alio manantium fluxum duci et cohiberi statu cardinis alicuius ab alio non manantis. 
llamamos Uno sería, en consecuencia, lo que hace que el alma sea movimiento y no un mero fluir. Y lo que permite que se distinga ella misma del cuerpo.

Así, el argumento del capítulo III concluye que la medida, la ratio para identificar las distinciones básicas, antes/después, superior/inferior, mejor/ peor, no puede encontrarse en el alma. Esa ratio no viene dada y es lo Uno, pues sólo desde algo que no emana ni fluye, es posible distinguir la diferencia en lo que emana y fluye. Finalmente, si el absoluto, lo uno, no puede ser fin de la vida moral, es el fundamento de toda cualificación, la fuente de toda distinción entre mejor y peor.

\section{ObSERVACIONES FINALES}

Las implicaciones más significativas de la introducción del argumento de la eminencia de la unidad en la Teología platónica de Ficino es que conduce a un distanciamiento del modelo teleológico aristotélico-tomista.

La razón es que para Ficino el alma no puede ver al Uno como su propio fin, porque no se le presenta como constituido de algún elemento común con ella misma, a semejanza de como ocurre en el modelo tomista en que cualquier ente se relaciona con Dios como fin en tanto que Dios mismo es ente perfecto, y por ende perfección de todo ente.

Al contrario, según se desprende de nuestro examen de la Teología platónica, el Uno se presenta al alma, a través de la mente, como razón de la distinción y, por tanto, de cualificación. En este sentido, el "reflejo" de la unidad en la mente es un acto reflexivo por el cual el alma toma conciencia de las diferencias, reconoce el mayor o menor bien en las cosas y, particularmente, toma conciencia de su diferencia con el cuerpo.

Hay que subrayar, sin embargo, que esta conciencia es un acto mediado por la mente y no una intuición del alma. Así, no sólo se subraya la distancia entre el Uno y el alma, sino que se muestra que la unidad, entendida como bien, tiene un carácter formal y no un contenido específico para el alma. En otras palabras, que es fundamento para valorar, distinguir y separar, pero no para identificar lo bueno como algo, un ser, una conducta, una esencia, porque a fin de cuentas de la unidad, como del bien, no puede decirse que sea algo.

De aquí se sigue que el distanciamiento del alma respecto al Uno signifique también que el alma se incline sobre el cuerpo y atienda sobre todo los fines perseguidos en la vida terrena, más urgentes e inmediatos para ella que cualquier otros y que estos no guarden una relación expresa con el Uno como fin último y sumo bien. 
Esta desarticulación de los fines de la vida y la unidad como fin, contrasta con lo que sostiene la tradición aristotélico-tomista, según la cual la forma de ciertas acciones en la vida terrena perfecciona la naturaleza del hombre y la ordena de acuerdo con un fin último. Así, la formalidad de la unidad —y la idea de fin y de bien dependiente de esta — hacen de la existencia humana en Ficino una búsqueda constante por reencontrar el lazo que une las acciones de la vida con la razón de la unidad de lo que existe y que pasa porque el alma misma se torne uno.

\section{Traducción}

\section{Marsilio Ficino, Teología platónica (acerca de la inmortalidad del alma)}

\section{Libro II, capítulo III:}

No hay tal número de dioses que uno esté por encima de otro eternamente

Probablemente alguien nos conceda que dios es superior al ángel; ${ }^{8}$ y que dios es uno. Así, ya que no pueden existir dioses iguales, sin embargo, puede existir un dios que esté por encima de otro a perpetuidad. Pensamos que, aunque ya fueron aducidos argumentos para refutar esto último, ${ }^{9}$ podemos hacerlo más claramente con otras razones.

${ }^{8}$ Esta superioridad del ángel está tomada del la Jerarquía celeste del Pseudo Dionisio: “Los teólogos dan el nombre de ángel' también a los órdenes más altos y santos de entre los seres celestes por el hecho de que manifiestan las iluminaciones procedentes de la Deidad" (196 B) (141).

${ }^{9}$ Se refiere aquí a los argumentos presentados en el capítulo II del Libro II de la Teología platónica. En él, Marsilio Ficino comienza por desechar la posibilidad de que existan dos principios iguales. No obstante procede a discutir qué pasaría si, en efecto, hubiera dos dioses iguales. El centro del argumento es que si los hubiera, estos serían, o completamente diferentes, o idénticos o parcialmente iguales. Desecha las primeras dos posibilidades porque, si fueran idénticos, sería el mismo, y si fueran completamente diferentes no podrían ser iguales. La tercera opción, el que fueran parcialmente similares, obliga, según Ficino, a asumir que tienen una naturaleza en común y, por tanto, que esa naturaleza en común es más alta que cualquiera de los dos principios que no serían simples sino compuestos — por la naturaleza común y la propia de cada uno-. Sobre esta base pasa a discutir los tres argumentos de los platónicos a favor de la existencia de un solo principio: que sería la máxima unidad, la máxima verdad y la máxima bondad. En el primero, el argumento es la existencia de un orden, la necesidad de un solo mandato para ese orden. Para los otros dos argumentos, el principio es semejante al presentado en relación con los dos principios. Se entiende pues, que en este capítulo III Ficino presentará un nuevo argumento a favor de la unidad.

Medievalia 50, 2018, pp. 247-260 
Si los dioses fueran innumerables, las causas de las cosas serían infinitas, y una causa estaría por encima de otra gradual y perpetuamente. Y si estos dioses innumerables son las causas de las cosas, también ellos estarían uno sobre otro gradual y perpetuamente. De esta forma, también los efectos de estas causas en el mundo serían innumerables, ya que cualquier causa produce algún efecto en el mundo. De esta manera, si una causa está por encima de otra, esto mismo sucedería con los efectos de esas causas. Por lo tanto, si las causas fueran infinitas y una superior a la otra, existirían también efectos inferiores, uno más deficiente que el otro. Y la causa superior siempre proyectaría su virtud más lejos en los efectos producidos que la de más abajo. De esta forma la proyección de los efectos sería al infinito si el ascenso de las causas fuera infinito. ${ }^{10}$

¿Quién no ve lo absurdo que es esto? En primer lugar, si en las cosas no existiera algún principio, no existiría ninguna consecuencia. Todas las cosas tienen un principio, ya que si no lo tuvieran, se negaría el fluir de todas las cosas; admitiendo que nunca tuvo comienzo tal fluir. Sabemos, también, que en el mundo hay cosas más perfectas que otras, y que crecen en virtud de la perfección al ascender, de la misma forma que decrecen al disminuir ésta. Esto quiere decir que la fuerza natural de las cosas se debilita gradualmente al descender. A partir de esto, podría pensarse que se deshaga completamente porque poco a poco disminuye. Precisamente por esto, una cosa no puede estar debajo de otra perpetuamente; así como tampoco pueden existir efectos infinitos.

No existen, por tanto, causas infinitas, debido a que al ascender, la perfección crece; esto se hará patente cuando hayamos alcanzado la felicidad suma. ¿Por qué consideramos al alma mejor que el cuerpo, si no es porque es más cercana a la suma bondad? Si no hubiera sitio para la bondad suma si no en el infinito, se iría ascendiendo de bondad en bondad, y el cuerpo estaría separado siempre de la suma bondad por un intervalo infinito, y lo mismo sucedería con el alma. Un infinito no es más amplio o más angosto que otro infinito; y nunca existirá una razón mesurada e ideal, por la que uno se prefiera al otro. Ya que nada es más cercano y propio al alma que la bondad suma, y no el cuerpo; y por tanto no habría nada mejor. Ni siquiera el ángel sería mejor que el alma. ${ }^{11}$

${ }^{10}$ Un argumento similar se encuentra en Proclo: "O bien se dará un proceso infinito, poniendo una causa detrás de otra, de forma que la afirmación de causas anteriores no acabará nunca” (Elementos de teología, 11, 34).

${ }^{11}$ Aquí Ficino continúa glosando el capítulo 11 de los Elementos de teología de Proclo. 
Así pues, cualquier cosa que fluya a partir de algo, lo hace por su propia naturaleza, porque nada hay después de ésta. Por un cierto flujo se consigue el ser. Si no existiera en las cosas un principio, cualquier cosa fluiría a partir de cualquier otra, y en consecuencia todas fluirían. Si esto fuera así, nunca existiría la unidad, la igualdad, la semejanza, la permanencia, el orden y la restitución. Sin embargo, porque estos atributos existen, es necesario que el flujo del cual manan las cosas sea conducido y contenido por un eje que no fluya. A semejanza del interminable flujo de los cuerpos líquidos que debe necesariamente tener un fin, y que no puede ser otro cuerpo líquido que fluya interminablemente, sino un cuerpo sólido.

Finalmente, un grado superior opera para uno inferior y del grado superior recibe algo. Si no existiera un primer grado en las cosas, no existiría un 256 grado último, ni siquiera un grado medio establecido a partir de los grados infinitos superiores. ${ }^{12}$ Precisamente por esto recibiría de los grados superiores excelencias infinitas y no recibiría nada de lo que genera su bondad, y las cosas inferiores representarían tesoros incuantificables, $y$, en verdad, cualquier cosa sería un manantial de riquezas. Si esto fuera así, tendrían infinitas virtudes y estarían llenas de perfecciones innumerables. Y de esta forma, todas las cosas serían similarmente infinitas. No existiría ninguna cosa mejor que otra, ni una causa mejor que su secuencia; o tal vez una cosa infinitamente finita, a causa de que sería superior a las consecuencias infinitas.

Nunca existiría una ciencia verdadera cuando las causas infinitas de las cosas no pueden ser comprendidas; y no existiría en el universo lo que impulsa o establece el apetito. Pues en virtud de esto, comienza y se establece todo el apetito.

Por tanto, uno sería absolutamente el principio de las cosas. Se le llama unidad, porque por su excelentísima simplicidad sobrepasa a todas las cosas; se le llama verdad, porque produciendo el ser lo da a todos; se le llama bondad, pone a disposición de sí las cosas producidas para ser conducidas hacia ella y el ser con bondad. Además, como transmite la teología de Juan el Apóstol, el más divino de todos los teólogos, al que acoge con gusto el platónico Amelio, la unidad es el principio, la verdad, la razón del principio, y la bondad, el principio del amor racional. ${ }^{13}$ Y se entiende que esta misma sustancia es la unidad buena y verdadera, o la verdad única y buena, o la bondad única y verdadera, y que Dios es único, bueno y verdadero. Y porque la unidad existe, existe la

${ }^{12}$ En todo este argumento Ficino parece seguir a Plotino en la Eneada sexta: "Las magnitudes continuas no tendrían razón de ser si no poseyesen la unidad” (381).

${ }^{13}$ La fuente de esta afirmación de Ficino es Eusebio de Cesarea, Praeparatio evangelica, 11, XIX, 540. 
verdad; y porque la unidad es buena, la bondad. Todas estas cosas están implícitas en la unidad, y explícitas en la verdad, y difundidas por la bondad. Sin embargo, después todas fluyen juntas; y refluyen por la bondad, adquieren una nueva forma por la verdad, y vuelven a ser una a causa de la unidad.

\section{Texto latino}

Marsilio Ficino. Teologia Platonica de Immortalitate Animorum Liber II Caput III

Non sunt dii plures, alius super alium sine fine.

Ceterum concedet forsitan nobis aliquis esse quidem deum super angelum, et unum esse deum, ita ut non sint dii plures aequales invicem, esse tamen deum alium super alium sine fine. Hoc licet arbitremur ex superioribus confutatum, aliis tamen rationibus clarius refellemus.

Si dii sic innumerabiles sint, erunt rerum causae infinitae se gradatim innumere superantes, siquidem ipsi dii rerum causae sunt et innumere alii aliispraeponuntur. Si ita, erunt quoque effectus in mundo innumerabiles. Quaelibet enim causa suum aliquem in rerum ordine producit effectum atque sicut causae se inter se superant ita superant et effectus. Igitur infiniti erunt effectus in descendendo, et innumere deficient sub se deinceps, si causae sint infinitae innumere se superantes. Et cum superior causa semper longius virtutem suam in producendis effectibus iaciat quam inferior, in infinitum erit iactus effectuum, si causarum fuerit infinitus ascensus.

Hoc quam absurdum sit, quis non viderit? Primo quidem, si non sit in rebusprimum aliquid, non erunt sequentia ulla. Cuneta enim trahuntur a primo, quod quidem si nusquam extet, non erit unde effluxus rerum aliunde fiuentium umquam exordiatur. Deinde videmus in rebus alias esse aliisperfectiores, perfectionisque virtutem ascendendo crescere, decrescere descendendo. Quo fit, ut vis naturalis rerum descendendo sensim debilitetur. Unde cogitur alicui omnino deficere, postquam minuitur paulatim. Ideoque non potest res alia esse sine fine sub alia. Itaque non erunt effectus innumeri. Non erunt igitur innumerae causae, siquidem eo ipso, quod ascendendo crescit perfiectio, perspicue constat illam quandoque ad summum venturam fiore. Cur iudicamus animam corpore meliorem, nisi quia sit summae bonitatipropinquior? Si nusquam esset bonitas summa, sed in infinitum de bono ascenderetur in bonum, per infinitum intervallum omnino distaret a summa bonitate corpus per infinitum similiter anima. Infinitum alterum non est aut amplius aut angustius altero infinito. Neque erit usquam ratio mensuraque idealis, per quam alia aliis praefierantur. Quare nihilo vel proponquior vel convenientior 
esset anima ipsi bonitati quam corpus, igitur neque melior; neque angelus melior anima.

Proinde quicquidfluit ab alio, natura suafluitat, quoniam et secundum se nihil est etperfluxum quendam prodit in esse. Si non sit in rebus primum aliquidy res quaelibet emanabit ab alia; omnes igiturfluitabunt. Quapropter nusquam erit unitas, aequalitas, similitudoy status, ordo et restitutio. Nunc vero quia haec rebus insunt, oportet rerum ab alio manantium fluxum duci et cohiberi statu cardinis alicuius ab alio non manantisy perinde ut iterminatum fluxum corporum liquidorum terminari necesse est, non alio corpore liquido similiter diffluente, sed corpore solido. Denique superior quilibet gradus in inferiorem aliquid operatur et a superiore accipit aliquid. Si nullus sit gradus in rebus primus nullusque sit ultimus, quilibet gradus medius a gradibus inflnitis superioribus dependebit, ac rursus gradus inflnitos producet inferiores. Quapropter accipiet a superioribusperfectiones innúmeras, cum accipiat a qualibet sui causa boni nonnihil; et inflnita muñera, inferioribus exhibebit, cuique enim aliquid largietur. Quapropter erit immensae virtutis et perfectionum plenus inflnitarum. Sic res omnes aeque erunt inflnitae. Non erit res alia praestantior alia, non erit causa suo opere melior. Vel forte res quaelibet innumere flnita, quia sequentia innumerabilia superávit. Nec erit usquam ulla vera scientia, cum nequeant inflnitae rerum causae comprehendi. Neque erit in universo quod apperirum moveat aut sistat, si non sit principium ultimusque flnis. Huius enim virtute movetur appetitus omnis atqueflrmatur.

Unum igitur omnino sit rerum principium. Vocetur unitas, quia per excellentissimam simplicitatem supereminet omnia; Veritas, quia producendo esse dat omnibus; bonitas, quia producta ad se revocando praestat et bene esse. Atque, ut tradit loannis Apostoli teologia, theologorum omnium divinissimi quem platonicus Amelius libenter amplecitur, unitatem vocato principium, veritatem principii rationem, bonitatem denique principii rationalis amorem. Atque haec ipsa substantia, scilicet unitas vera bona, seu Veritas una bona, sive bonitas una vera, sit unus bonus verusque deus. Sed quia unitas est, ideo sit Veritas; quia unitas vera, ideo bonitas. In unitate implicat cuncta explicat in veritate, effunditper bonitatem. Cuncta vero postquam inde fluxerunt, refluuntper bonitatem, reformantur per veritatem, restituuntur in unum per unitatem.

\section{Comentario al TeXto latino}

En su célebre Carta a Ermolao Barbaro, Giovanni Pico della Mirandola deja un precioso testimonio de lo que en esa época de efervescencia intelectual era la filosofía y la escritura filosófica, por un lado, y la retórica y el estilo, por 
el otro. Al parecer en el Renacimiento se le exigía al filósofo, naturalmente, hacer filosofía pero también hacer literatura.

Giovanni Pico se demuestra como un escritor versátil, además de auténtico filósofo; aunque cultiva por convicción el "estilo parisino", es decir, el latín medieval — "bárbaro", austero, inculto — formado originalmente por el postulado de que la verdad no necesita de adornos para ser expresada. En el caso de Marsilio Ficino y su Teología platónica, nos encontramos ante un escritor que pudo remontar, por sus amplios conocimientos de la lengua griega y latina, las influencias inmediatas de su pensamiento - San Agustín, Santo Tomás, Pseudo Dionisio Aeropagita - y alimentarse directamente de las fuentes - Platón, Aristóteles, los Himnos órficos, Plotino, Lucrecio, Cicerón, Séneca-.

$\mathrm{Al}$ hacer esto, Ficino aprehendió también los complejos fundamentos de la retórica clásica, que involucraban necesariamente la belleza en la expresión — qué hay más literario que los diálogos platónicos-, es decir, estar preocupado por dotar a la frase de un cierto ritmo, evitar aliteraciones y cacofonías y, en la medida de lo posible, incorporar schemata — figuras retóricas- al discurso.

Notamos una sutil intención estilística en la escritura de la Teología platónica. Un filósofo con las características de Ficino evitará, obviamente, el uso de "figuras de pensamiento", pero no de las de dicción. Ficino no gusta de usar la misma palabra en frases contiguas, opta siempre por un sinónimo. Así, en el capítulo tercero del libro segundo, encontramos que el adjetivo infinitus - a -um se sustituye por innumerabilis -e, innumerus - a-um, o la frase sine fine; y el comparativo superior, por praestantior o melior.

Por lo demás, la sintaxis de Ficino oscila entre el latín clásico y el latín medieval, incorporando, de vez en vez, ciertos giros exclusivos de la lengua griega. Estas son las “irregularidades” más comunes en el latín ficiniano, según Michele Schiavone:

Interrogativas indirectas sin subjuntivo; an non en lugar de necne en las interrogativas indirectas y necne en lugar de an non en las interrogativas directas; sequitur quod con indicativo; sequitur con acusativo e infinitivo; quod con subjuntivo en lugar de ut con subjuntivo; falta de cuidado en el uso de la atracción modal; idem en lugar de ipse, nullus en lugar de nemo; sicut ... sic o sic... sicut en lugar de ut... sic o sic... ut; aliquis en lugar de quisquam; ipse concordando con el antecedente y el sujeto; preposiciones sobrentendidas; impartio por impertio; uso de barbarismos lexicales como sibi por ei y ei por sibi; etiam en lugar de quoque; debeo con infinitivo en lugar de la perifrástica pasiva; aliquis después de ne, num, nisi, nonne; per con acusativo en lugar de ablativo de instrumento; neque... quidem en lugar de 
ne... quidem; los casos oblicuos del pronombre neutro formados en femenino en lugar de neutro al acompañar a la palabra res; los ablativos de la tercera declinación en - $i$ y - $e$ son usados indiscriminadamente; -que enclítico seguido del quoque enfático; alii en lugar de ceteri; namque como enclítico; perdor en lugar de pereo; quando en lugar de cum; aliquid en lugar de aliquod; tantum abest quod con subjuntivo en lugar de $u$ t; uso indiscriminado de intellego e intelligo; ratio ob quam con subjuntivo en lugar de ratio cur ("Introduzione", 30-31).

\section{BiBLIOGRAFÍA}

Allen, Michael J. B., Icastes: Marsilio Ficino's Interpretation of Plato's Sophist, Los Ángeles: University of California Press, 1984.

Aquino Tomás, El ente y la esencia, trad. de Manuel Fuentes Benot, Buenos Aires: Aguilar, 1981.

Aquino Tomás,, Cuestión disputada sobre las virtudes en general, estudio preliminar, traducción y notas, Laura E. Corso de Estrada, Pamplona: Eunsa, 2000.

Ficino, Marsilio, Platonic Theology, trad. de Michael J. B. Allen con John Warden, London: Harvard University Press, 2001.

Ficino, Marsilio, Sobre el amor. Comentario al Banquete de Platón, trad. de Maria Pia Lamberti y José Luis Bernal, México: Universidad Nacional Autónoma de México, 1994.

Ficino, Marsilio, Teologia platonica, trad. de Michele Schiavone, Bologna: Centro di studi filosofici di Gallarte, 1965.

Garín, Eugenio. Marsilio Ficino y el platonismo, trad. de Ariela Battán, Buenos Aires: Alicón, 1997.

Kristeller, Paul Oscar, Il pensiero filosofico di Marsilio Ficino, Firenze: Sansoni, 1953.

Platón, Diálogos III (Fedón, Banquetes, Fedro), traducciones de Carlos García Gual, M. Martínez Hernández y Emilio Lledó, Madrid: Gredos, 1988.

Plotino, Eneada sexta, traducción, prólogo y notas Francisco de P. Samaranch, Buenos Aires: Aguilar, 1975.

Proclo, Elementos de teología, traducción, prólogo y notas Francisco de P. Samaranch, Buenos Aires: Aguilar, 1965.

Proclo, Teología platónica, trad, de Michele Abbate, Milano: Bompiani, 2005.

Pseudo Dionisio Aeropagita, “Jerarquía celeste”, en Obras completas, edición preparada por Teodoro H. Martín, Madrid: Biblioteca de Autores Cristianos, 1990.

Schiavone, Michele, “Introduzione”, en Marsilio Ficino, Teologia platonica, trad. de Michele Schiavone, Bologna: Centro di studi filosofici di Gallarte, 1965. 